Research Article

\title{
Blow-Up of Certain Solutions to Nonlinear Wave Equations in the Kirchhoff-Type Equation with Variable Exponents and Positive Initial Energy
}

\author{
Loay Alkhalifa $\mathbb{D}^{1},{ }^{1}$ Hanni Dridi, ${ }^{2}$ and Khaled Zennir $\mathbb{D}^{1,3}$ \\ ${ }^{1}$ Department of Mathematics, College of Sciences and Arts, Qassim University, Ar Rass, Saudi Arabia \\ ${ }^{2}$ Laboratory of Applied Mathematics, Badji Mokhtar University, P.O. Box 12, 23000 Annaba, Algeria \\ ${ }^{3}$ Laboratoire de Mathématiques Appliquées et de Modélisation, Université 8 Mai 1945 Guelma, B.P. 401 Guelma 24000, Algeria \\ Correspondence should be addressed to Loay Alkhalifa; loay.alkhalifa@qu.edu.sa
}

Received 2 March 2021; Revised 16 March 2021; Accepted 22 March 2021; Published 8 April 2021

Academic Editor: Liliana Guran

Copyright (C) 2021 Loay Alkhalifa et al. This is an open access article distributed under the Creative Commons Attribution License, which permits unrestricted use, distribution, and reproduction in any medium, provided the original work is properly cited.

This paper is concerned with the blow-up of certain solutions with positive initial energy to the following quasilinear wave equation: $u_{t t}-\mathscr{M}(\mathcal{N} u(t)) \Delta_{p(\cdot)} u+g\left(u_{t}\right)=f(u)$. This work generalizes the blow-up result of solutions with negative initial energy.

\section{Introduction}

Let $\Omega$ be an open bounded Lipschitz domain in $\mathbb{R}^{n}(n \geq 1)$, $T>0, Q_{T}=\Omega \times(0, T)$. We consider the following nonlinear hyperbolic equation:

$$
\begin{cases}u_{t t}-\mathscr{M}(\mathcal{N} u(t)) \Delta_{p(x)} u+g\left(u_{t}\right)=f(u), & (x, t) \in Q_{T}, \\ u(x, t)=0, & x \in \partial \Omega, t \in(0, T), \\ u(x, 0)=u_{0}(x), u_{t}(x, 0)=u_{1}(x), & x \in \Omega .\end{cases}
$$

Here, $\partial \Omega$ is a Lipschitz continuous boundary. The initial conditions meet the following:

$$
\begin{aligned}
& u_{0} \in W_{0}^{1, p(\cdot)}(\Omega), \\
& u_{1} \in L^{2}(\Omega) .
\end{aligned}
$$

The Kirchhoff function $\mathscr{M}: \mathbb{R}^{+} \longrightarrow \mathbb{R}^{+}$is continuous and has the standard form:

$$
\mathscr{M}(\tau)=a+b \gamma \tau^{\gamma-1}, \quad a, b \geq 0, \gamma \geq 1, a+b>0, \gamma>1 \text { if } b>0 .
$$

The elliptic nonhomogeneous $p(x)$-Laplacian operator is defined by

$$
\Delta_{p(x)} u=\nabla \cdot\left(|\nabla u|^{p(x)-2} \nabla u\right)
$$

where $\nabla \cdot$ is the vectorial divergence and $\nabla$ is the gradient of $u$. The functional

$$
\mathscr{N} u(t)=\int_{\Omega} \frac{|\nabla u|^{p(x)}}{p(x)} d x
$$

is the naturally associated $p(x)$-Dirichlet energy integral. The term with a variable exponent

$$
f(u)=c(x, t)|u|^{q(x)-2} u,
$$

plays the role of a source, and the dissipative term with a variable exponent

$$
g\left(u_{t}\right)=d(x, t)\left|u_{t}\right|^{r(x)-2} u_{t},
$$

is a strong damping term. 
The coefficients $c$ and $d$ are continuous in $Q_{T}$ and satisfy

$0<c^{-}=\inf _{(x, t) \in Q_{T}} c(x, t) \leq c(x, t) \leq c^{+}=\sup _{(x, t) \in Q_{T}} c(x, t)<\sigma<\infty$,

$0<d^{-}=\inf _{(x, t) \in Q_{T}} d(x, t) \leq d(x, t) \leq d^{+}=\sup _{(x, t) \in Q_{T}} d(x, t)<\infty$,

where $\sigma$ is a constant defined in (38). We assume that the Kirchhoff function $\mathscr{M}$, defined by (3), satisfies the following hypotheses:

(i) For $1<\alpha \leq \beta<\min \left\{n / p^{+}, n p^{-} / p^{+}\left(n-p^{-}\right)\right\}$, there exist $m_{2} \geq m_{1}>0$ such that

$$
m_{1} \tau^{\alpha-1} \leq \mathscr{M}(\tau) \leq m_{2} \tau^{\beta-1}, \quad \tau \in \mathbb{R}^{+}
$$

(ii) For all $\tau \in \mathbb{R}^{+}$, it holds that

$$
\int_{0}^{\tau} \mathscr{M}(s) d s=\hat{M}(\tau) \geq \mathscr{M}(\tau) \tau .
$$
satisfy

The exponents $p(\cdot), q(\cdot)$, and $r(\cdot)$ are continuous and

$$
\begin{aligned}
2 & \leq \min \left\{p^{-}, r^{-}\right\} \leq\{p(x), r(x)\} \leq \max \left\{p^{+}, r^{+}\right\}<q^{-} \\
& \leq q(x) \leq q^{+}<p^{-} \alpha \leq p^{-} \beta \leq p^{+} \alpha \leq p^{+} \beta \leq p_{*}(x),
\end{aligned}
$$

where the constants $\alpha$ and $\beta$ are given in (10) and

$$
\begin{aligned}
& p^{-}=\text {ess } \inf _{x \in \Omega} p(x), \\
& p^{+}=\text {ess } \sup _{x \in \Omega} p(x), \\
& q^{-}=\text {ess } \inf _{x \in \Omega} q(x), \\
& q^{+}=\text {ess } \sup _{x \in \Omega} q(x), \\
& r^{-}=\text {ess } \inf _{x \in \Omega} r(x), \\
& r^{+}=\text {ess } \sup _{x \in \Omega} r(x) .
\end{aligned}
$$

Also, we can define $p_{*}(x)$ by

$$
p_{*}(x)= \begin{cases}\frac{n p(x)}{\operatorname{ess} \sup _{x \in \Omega}(n-r(x))}, & \text { if } p^{+}<n, \\ +\infty, & \text { if } p^{+} \geq n .\end{cases}
$$

We also assume that $p(\cdot), q(\cdot)$, and $r(\cdot)$ satisfy the logHölder continuity condition

$$
|\xi(x)-\xi(y)| \leq-\frac{L}{\log |x-y|}, \quad \text { for a.e., } x, y \in \Omega,|x-y|<\delta,
$$

for $L>0,0<\delta<1$.

Problem (1) models several physical and biological systems such as viscoelastic fluids, filtration processes through a porous medium, and fluids with viscosity dependent on temperature. In the intention of problem (1), we can see that it is linked to the following equation presented by Kirchhoff and Hensel [1] in 1883:

$$
\rho \frac{\partial^{2} u}{\partial t^{2}}-\left(\frac{P_{0}}{h}+\frac{E}{2 L} \int_{0}^{L}\left|\frac{\partial u}{\partial x}\right| d x\right) \frac{\partial^{2} u}{\partial x^{2}}+g\left(u_{t}\right)=f(u) .
$$

The parameters $L, h, E, \rho$, and $P_{0}$ represent, respectively, the length of the string, the area of the cross-section, Young's modulus of the material, the mass density, and the initial tension. This equation is an extension of the classic d'Alembert's wave equation by looking at the effects of changes in the length of the string during the vibrations. As for this problem, it has been studied. More precisely, for $g\left(u_{t}\right)=u_{t}$, the global existence and nonexistence results can be found in $[2,3]$, and for $g\left(u_{t}\right)=\left|u_{t}\right|^{p} u_{t}, p>0$, the main results of existence and nonexistence are in the paper [4]. In recent years, hyperbolic problems with a constant exponent have been studied by many authors; we refer to interesting works [57]. However, only a little research has been done regarding hyperbolic problems with nonlinearities of the variable exponent type; some interesting works can be found in [8-13].

Recently, in [14], Piskin studied the following wave equation with variable exponent nonlinearities:

$$
u_{t t}-M\left(\|\nabla u\|^{2}\right) \Delta u+\left|u_{t}\right|^{p(x)-2} u_{t}=|u|^{q(x)-2} u .
$$

The author proved, by using the modified energy functional method, the existence of solutions. We have also looked at the asymptotic behavior of the Kirchhoff wave equation problems. We can say that the investigation into the determination of the type, as well as the rate of decay, was the focus of attention of many researchers whose work was represented in $[15,16]$. Motivated by previous studies, in this work, we consider problem (1), which is more interesting and applicable in the real approach of sciences, so a finite-time blow-up for certain solutions with positive and also negative initial energy has been proved. More precisely, our aim here is to find sufficient conditions on the variable exponents $p(\cdot), q(\cdot)$, and $r(\cdot)$ and the initial data for which the blow-up occurs. This paper is organized as follows. After the introduction in the first section, we will give some preliminaries in Section 2. Then, in Section 3, we state the main results which will be proved in Sections 4 and 5 . 


\section{Preliminaries}

Regarding some definitions and basic properties of the generalized Lebesgue-Sobolev spaces $L^{p(x)}(\Omega)$ and $W^{1, p(x)}(\Omega)$, where $\Omega$ is an open subset of $\mathbb{R}^{n}$, we refer to the book of Musielak [17] and the papers [18, 19]. Let

$$
C_{+}(\bar{\Omega}):=\{h: h \in C(\bar{\Omega}), h(x)>1, \quad \text { for all } x \in \bar{\Omega}\} .
$$

For any $h \in C(\bar{\Omega})$, we write

$$
\begin{aligned}
& h^{+}=\sup _{x \in \Omega} h(x), \\
& h^{-}=\inf _{x \in \Omega} h(x) .
\end{aligned}
$$

Then, for any $p(x) \in C^{+}(\bar{\Omega})$, we define the variable exponent Lebesgue space as follows:

$L^{p(\cdot)}(\Omega)=\left\{u: \Omega \longrightarrow \mathbb{R}\right.$ measurable $: \varrho_{p(\cdot)}(\mu u)<+\infty, \quad$ for some $\left.\mu>0\right\}$,

where $\varrho_{p(\cdot)}$ is the $p(\cdot)$ modular of $u$, and it is defined by

$$
\mathrm{\varrho}_{p(\cdot)}(u)=\int_{\Omega}|u|^{p(x)} d x .
$$

It is equipped with the following so-called Luxemburg norm on this space defined by the formula

$$
\|u\|_{p(\cdot)}=\inf \left\{\mu>0: \int_{\Omega}\left|\frac{u(x)}{\mu}\right|^{p(x)} d x \leq 1\right\} .
$$

Variable exponent Lebesgue spaces resemble classical Lebesgue spaces in many aspects: they are Banach spaces, the Hölder inequality holds, they are reflexive if and only if $1<p^{-} \leq p^{+}<\infty$, and their continuous functions are dense if $p^{+}<\infty$.

Lemma 1. Suppose that $p(\cdot)$ satisfies (15); then,

$$
\|u\|_{p(\cdot)} \leq C\|\nabla u\|_{p(\cdot)}, \quad \forall u \in W_{0}^{1, p(\cdot)}(\Omega),
$$

where $C>0$ is a constant that depends only on $p^{-}, p^{+}$, and $\Omega$.

Lemma 2. If $p(\cdot) \in C(\bar{\Omega})$ and $q: \Omega \longrightarrow[1, \infty)$ are measurable functions such that

$$
\text { ess } \inf _{x \in \Omega}\left(p_{*}(x)-q(x)\right)>0, \quad \text { with } p_{*}(x)= \begin{cases}\frac{n p(x)}{\text { ess } \sup _{x \in \Omega}(n-p(x))}, & \text { if } q^{-}<n, \\ \infty, & \text { if } q^{-} \geq n,\end{cases}
$$

then the embedding $W_{0}^{1, p(\cdot)}(\Omega)^{\circ} L^{q(\cdot)}(\Omega)$ is continuous and compact.
Lemma 3. Let $1<p^{-} \leq p^{+}<+\infty$. The spaces $L^{p(\cdot)}(\Omega)$ and $W^{1, p(\cdot)}(\Omega)$ are separable, uniformly convex, and reflexive Banach spaces. The conjugate space of $L^{p(\cdot)}(\Omega)$ is $L^{p^{\prime}(\cdot)}(\Omega)$, where

$$
\frac{1}{p(x)}+\frac{1}{p^{\prime}(x)}=1, \quad \forall x \in \Omega .
$$

For $u \in L^{p(\cdot)}(\Omega)$ and $v \in L^{p^{\prime}(\cdot)}(\Omega)$, we have

$$
\left|\int_{\Omega} u(x) v(x) d x\right| \leq\left(\frac{1}{p^{-}}+\frac{1}{\left(p^{\prime}\right)^{-}}\right)\|u\|_{p(\cdot)}\|v\|_{p}^{\prime(\cdot)} .
$$

Lemma 4. If $p \geq 1$ is a measurable function on $\Omega$ and $u \in$ $L^{p(\cdot)}(\Omega)$, then $\|u\|_{p(\cdot)} \leq 1$ and $\rho_{p(\cdot)}(u) \leq 1$ are equivalent. For $u \in L^{p(\cdot)}(\Omega)$, we have

$$
\begin{aligned}
& \|u\|_{p(\cdot)} \leq 1 \text { implies } \rho_{p(\cdot)}(u) \leq\|u\|_{p(\cdot)}, \\
& \|u\|_{p(\cdot)}>1 \text { implies } \rho_{p(\cdot)}(u) \geq\|u\|_{p(\cdot)} .
\end{aligned}
$$

Lemma 5. If $p(x) \in[1, \infty)$ is a measurable function on $\Omega$, then

$$
\min \left\{\|u\|_{p(\cdot)}^{p^{-}},\|u\|_{p(\cdot)}^{p^{+}}\right\} \leq \rho_{p(\cdot)}(u) \leq \max \left\{\|u\|_{p(\cdot)}^{p^{-}}\|u\|_{p(\cdot)}^{p^{+}}\right\},
$$

for all $u \in L^{p(\cdot)}(\Omega)$.

\section{Main Results}

Now, we state without proof the following existence result.

Proposition 6. Assume that (2) holds and the coefficients $a, b$, $c$, and $d$ satisfy (3) and (9) and the exponents $p, q$, and $r$ satisfy (12). Then, problem (1) has a unique weak solution such that

$$
\left\{\begin{array}{l}
u \in L^{\infty}\left((0, T), W_{0}^{1, p(\cdot)}(\Omega)\right), \\
u_{t} \in L^{\infty}\left((0, T), L^{2}(\Omega)\right), \\
u_{t t} \in L^{\infty}\left((0, T), W_{0}^{-1, p^{\prime}(\cdot)}(\Omega)\right),
\end{array}\right.
$$

where $p^{\prime}(\cdot)$ is the conjugate exponent of $p(\cdot)$.

Remark 7. The proof can be established by employing the Galerkin method as in the work of Antontsev [8].

We first define the energy function. Let

$$
\mathscr{E}(t):=\frac{1}{2}\left\|u_{t}\right\|_{L^{2}(\Omega)}^{2}+\widehat{M}(\mathcal{N} u(t))-\Psi(t),
$$


where

$$
\begin{aligned}
\widehat{\mathscr{M}}(\mathcal{N} u(t)) & =a \mathcal{N} u(t)+b \gamma[\mathcal{N} u(t)]^{\gamma}, \\
\Psi(t) & =\int_{\Omega} \frac{c(x, t)}{q(x)}|u|^{q(x)} d x .
\end{aligned}
$$

In order to investigate the properties of $\mathscr{E}(t)$, the following lemma is necessary.

Lemma 8. Suppose that $u$ is a solution of problem (1) that satisfies (29); then, we have

$$
\mathscr{E}_{t}(t)=-\int_{\Omega} \frac{c_{t}(x, t)}{q(x)}|u|^{q(x)} d x-\int_{\Omega} d(x, t)\left|u_{t}\right|^{r(x)} d x .
$$

Proof. By using the energy function (30) and problem (1), we directly deduce (32).

We also introduce the following lemma.

Lemma 9. Suppose that the conditions of Lemmas 1-5 hold. Then, there exists a constant $C>1$, which is a generic constant that depends on $\Omega$ only, such that

$$
\mathrm{\varrho}_{q(\cdot)}^{s / q^{-}}(u) \leq C\left(\|\nabla u\|_{p(\cdot)}^{\alpha p^{-}}+\mathrm{Q}_{q(\cdot)}(u)\right),
$$

for any $u \in W_{0}^{1, p(\cdot)}(\Omega)$ and $\alpha p^{-} \leq s \leq \alpha q^{-}$.

Proof. If $\mathrm{Q}_{q(\cdot)}(u)>1$, then

$$
\mathrm{e}_{q(\cdot)}^{s / q^{-}}(u) \leq \mathrm{Q}_{q(\cdot)}^{\alpha}(u) \leq C\left(\|\nabla u\|_{p(\cdot)}^{\alpha p^{-}}+\mathrm{Q}_{q(\cdot)}(u)\right) .
$$

If $\mathrm{Q}_{q(\cdot)}(u) \leq 1$, then we deduce by Lemma 4 that $\|u\|_{q(\cdot)}$ $\leq 1$. Then, Lemmas 2 and 5 imply

$$
\begin{aligned}
\mathrm{e}_{q(\cdot)}^{s / q^{-}}(u) & \leq \mathrm{e}_{q(\cdot)}^{\alpha p^{-} / q^{-}}(u) \leq \max \left\{\|u\|_{q(\cdot)}^{q^{-}},\|u\|_{q(\cdot)}^{q^{+}}\right\}^{\alpha p^{-} / q^{-}} \\
& =\|u\|_{q(\cdot)}^{\alpha p^{-}} \leq C\|\nabla u\|_{p(\cdot)}^{\alpha p^{-}} .
\end{aligned}
$$

Let $B$ be the best constant of the Sobolev embedding

$$
W_{0}^{1, p(\cdot)^{\circ}} L^{q(\cdot)}
$$

We set

$$
\begin{aligned}
& B_{1}=\max \left\{1, B,\left(\frac{m_{1}}{c^{+} \alpha\left(p^{+}\right)^{\alpha-1}}\right)^{1 / \alpha p^{+}}\right\} \\
& \sigma=\min \left\{1,\left(\frac{q^{-} m_{1}}{B_{1}^{q^{-}} \alpha\left(p^{+}\right)^{\alpha}}\right) \min \left\{\Gamma_{1}^{1-\left(q^{-} / \alpha p^{+}\right)}, \Gamma_{1}^{1-\left(q^{+} / \alpha p^{+}\right)}\right\}\right\}
\end{aligned}
$$

$$
\begin{aligned}
& \Gamma_{1}=\left(\frac{m_{1}\left(p^{+}\right)^{1-\alpha}}{c^{+} B_{1}^{q^{-}}}\right)^{\alpha p^{+} /\left(q^{-}-\alpha p^{+}\right)}, \\
& \mathscr{E}_{1}=\left[\frac{m_{1}\left(p^{+}\right)^{-\alpha}}{q^{-} \alpha}\left(q^{-}-p^{+}\right)\right] \Gamma_{1} .
\end{aligned}
$$

Now, the main results of the blow-up for certain solutions with positive/negative initial energy are given by the following theorems.

Theorem 10. Let the assumptions of Proposition 6 be satisfied, and assume that

$$
\begin{aligned}
& c^{+}<\sigma, c_{t}(x, t) \geq \tilde{\sigma} \geq 0, \quad \forall(x, t) \in Q_{T}, \\
& 0<\mathscr{E}(0)<\mathscr{E}_{1}, \quad \Gamma_{1}<\Gamma_{0}=\left\|\nabla u_{0}\right\|_{p(\cdot)}^{\alpha p^{+}} \leq B_{1}^{-\alpha p^{+}} .
\end{aligned}
$$

Then, the solutions of (1) blow up in finite time:

$$
T^{*} \leq \frac{1-\lambda}{\mu \lambda \mathscr{F}^{\lambda /(1-\lambda)}(0)}, \quad \lambda, \mu>0 .
$$

Theorem 11. Let the assumptions of Proposition 6 be satisfied, and assume that

$$
\mathscr{E}(0)<0
$$

Then, the solution of (1) blows up in finite time (42).

\section{Proof of Theorem 10}

To prove Theorem 10, we need the following lemmas.

Lemma 12. Let the assumptions of Theorem 10 hold; then, there exists $\sigma>0$ such that for any $c^{+}<\sigma$, there exist a constant $\Gamma_{2}>\Gamma_{1}$ such that

$$
\|\nabla u(\cdot, t)\|_{p(\cdot)}^{\alpha p^{+}} \geq \Gamma_{2}, \quad \forall t \geq 0 .
$$

Proof. By using the hypothesis (10) and the function (30), we obtain

$$
\begin{aligned}
\mathscr{E}(t) \geq & \hat{M}(t)-\Psi(t) \geq \frac{m_{1}}{\alpha\left(p^{+}\right)^{\alpha}} \rho_{p(\cdot)}^{\alpha}(\nabla u)-\frac{c^{+}}{q^{-}} \rho_{q(\cdot)}(u) \\
\geq & \frac{m_{1}}{\alpha\left(p^{+}\right)^{\alpha}} \min \left\{\|\nabla u\|_{p(\cdot)}^{\alpha p^{-}},\|\nabla u\|_{p(\cdot)}^{\alpha p^{+}}\right\} \\
& -\frac{c^{+}}{q^{-}} \max \left\{\|u\|_{q(\cdot)}^{q^{-}},\|u\|_{q(\cdot)}^{q^{+}}\right\} \\
\geq & \frac{m_{1}}{\alpha\left(p^{+}\right)^{\alpha}} \min \left\{\|\nabla u\|_{p(\cdot)}^{\alpha p^{-}},\|\nabla u\|_{p(\cdot)}^{\alpha \alpha^{+}}\right\} \\
& -\frac{c^{+}}{q^{-}} \max \left\{\left(B_{1}\|\nabla u\|_{p(\cdot)}\right)^{q^{-}},\left(B_{1}\|\nabla u\|_{p(\cdot)}\right)^{q^{+}}\right\} \\
= & \frac{m_{1}}{\alpha\left(p^{+}\right)^{\alpha}} \min \left\{\Gamma^{p^{-} / p^{+}}, \Gamma\right\} \\
& -\frac{c^{+}}{q_{-}} \max \left\{\left(B_{1}^{\alpha p^{+}} \Gamma\right)^{q^{q^{\prime}} / \alpha p^{+}},\left(B_{1}^{\alpha \alpha^{+}} \Gamma\right)^{q^{+} / \alpha p^{+}}\right\} \\
:= & \psi(\Gamma), \quad \forall \Gamma \in \mathbb{R}^{+},
\end{aligned}
$$


where $\Gamma=\|\nabla u\|_{p(\cdot)}^{\alpha p^{+}}$. Let the function

$$
\phi:[0,1] \longrightarrow \mathbb{R},
$$

be defined by

$$
\phi(\Gamma)=\frac{m_{1}}{\alpha\left(p^{+}\right)^{\alpha}} \Gamma-\frac{c^{+}}{q^{-}}\left(B_{1}^{\alpha p^{+}} \Gamma\right)^{q^{-} / \alpha p^{+}} .
$$

Notice that $\phi(\Gamma)=\psi(\Gamma)$, for $0<\Gamma \leq B_{1}^{-\alpha p^{+}}$. It is easy to check that the function $\phi$ is increasing for $0<\Gamma<\Gamma_{1}$ and decreasing for $\Gamma_{1}<\Gamma \leq \infty$. On the other hand, by (38), we deduce that, for any $c^{+}<\sigma$, since $\mathscr{E}(0)<\mathscr{E}_{1}=\phi\left(\Gamma_{1}\right)$, there exists a positive constant $\Gamma_{2} \in\left(\Gamma_{1}, \infty\right)$ such that $\phi\left(\Gamma_{2}\right)=\mathscr{E}(0)$. Then, we have $\phi\left(\Gamma_{0}\right)=\psi\left(\Gamma_{0}\right) \leq \mathscr{E}(0)=\phi\left(\Gamma_{2}\right)$. This implies that $\Gamma_{0} \geq \Gamma_{2}$.

Now, we suppose on the contrary that $\left\|\nabla u\left(t_{0}\right)\right\|_{p(\cdot)}^{\alpha p^{+}}<\Gamma_{2}$ for some $t_{0}>0$. Then, there exists $t_{1}>0$ such that $\Gamma_{1}<$ $\left\|\nabla u\left(t_{1}\right)\right\|_{p(\cdot)}^{\alpha p^{+}}$. Using the monotonicity of $\phi(\Gamma)$, we have

$$
\mathscr{E}\left(t_{1}\right) \geq \phi\left(\left\|\nabla u\left(t_{1}\right)\right\|_{p(\cdot)}^{\alpha p^{+}}\right)>\phi\left(\Gamma_{2}\right)=\mathscr{E}(0),
$$

which contradicts $\mathscr{E}(t)<\mathscr{E}(0)$, for all $t \in(0, T)$.

Lemma 13. Let the assumptions of Theorem 10 hold. Then, in light of Lemma 12, we have

$$
\rho_{q(\cdot)}(u) \geq \kappa, \quad \kappa>0
$$

Proof. By using (30), we get

$$
\begin{aligned}
\frac{c^{+}}{q^{-}} \rho_{q(\cdot)}(u) & \geq \Psi(t) \geq-\mathscr{E}(0)+\frac{m_{1}}{\alpha\left(p^{+}\right)^{\alpha}} \rho_{p(\cdot)}^{\alpha}(\nabla u)+\frac{1}{2}\left\|u_{t}\right\|_{L^{2}(\Omega)}^{2} \\
& \geq \frac{m_{1}}{\alpha\left(p^{+}\right)^{\alpha}} \rho_{p(\cdot)}^{\alpha}(\nabla u)-\psi\left(\Gamma_{2}\right) \\
& \geq \frac{c^{+}}{q_{-}} \max \left\{\left(B_{1}^{\alpha p^{+}} \Gamma_{2}\right)^{q^{-} / \alpha p^{+}},\left(B_{1}^{\alpha p^{+}} \Gamma_{2}\right)^{q^{+} / \alpha p^{+}}\right\} \\
& :=\kappa .
\end{aligned}
$$

Let

$$
\mathscr{H}(t)=\mathscr{E}_{1}-\mathscr{E}(t)
$$

Lemma 14. Let the assumptions of Theorem 10 be satisfied; then, we have

$$
0<\mathscr{H}(0) \leq \mathscr{H}(t) \leq \frac{\sigma}{q^{-}} \rho_{q(\cdot)}(u)
$$

Proof. Using (30), (32), and (51), we obtain

$$
0<\mathscr{H}(0) \leq \mathscr{H}(t) \leq \mathscr{E}_{1}-\frac{1}{2}\left[\left\|u_{t}\right\|_{L^{2}(\Omega)}^{2}+\widehat{M}(\mathscr{N} u(t))\right]+\Psi(t)
$$

Then, the use of (10) gives

$$
\begin{aligned}
\mathscr{E}_{1} & -\left[\frac{1}{2}\left\|u_{t}\right\|_{L^{2}(\Omega)}^{2}-\hat{\mathscr{M}}(\mathcal{N} u(t))\right] \leq \mathscr{E}_{1}-\int_{0}^{\mathcal{N} u(t)} \mathscr{M}(\tau) d \tau \\
& \leq \mathscr{E}_{1}-\frac{m_{1}}{\alpha\left(p^{+}\right)^{\alpha}} \min \left\{\|\nabla u\|_{p(\cdot)}^{\alpha p^{-}},\|\nabla u\|_{p(\cdot)}^{\alpha p^{+}}\right\} \\
& \leq \mathscr{E}_{1}-\frac{m_{1}}{\alpha\left(p^{+}\right)^{\alpha}} \min \left\{\Gamma_{2}^{p^{-} / p^{+}}, \Gamma_{2}\right\} \\
& \leq \mathscr{E}_{1}-\frac{m_{1}}{\alpha\left(p^{+}\right)^{\alpha}} \min \left\{\Gamma_{1}^{p^{-} / p^{+}}, \Gamma_{1}\right\} \\
& =\mathscr{E}_{1}-\frac{m_{1}}{\alpha\left(p^{+}\right)^{\alpha}} \Gamma_{1} .
\end{aligned}
$$

Now, recalling $\mathscr{E}_{1}$ in (38), we have

$\mathscr{E}_{1}-\left[\frac{1}{2}\left\|u_{t}\right\|_{L^{2}(\Omega)}^{2}-\widehat{\mathscr{M}}(\mathcal{N} u(t))\right] \leq-\frac{m_{1}\left(p^{+}\right)^{1-\alpha}}{q^{-} \alpha} \Gamma_{1}<0$.

On the other hand, we use (9) to get

$$
\Psi(t) \leq \frac{c^{+}}{q^{-}} \rho_{q(\cdot)}(u) \leq \frac{\sigma}{q^{-}} \rho_{q(\cdot)}(u)
$$

Combining (55) with (56) gives (52).

Corollary 15. Under the assumptions of Lemma 9, we have
(i) $\|u\|_{q^{-}}^{s} \leq C\left(\|\nabla u\|_{p(\cdot)}^{\alpha p^{-}}+\|u\|_{q^{-}}^{q^{-}}\right)$
(ii) $\rho_{q(\cdot)}^{s / q^{-}} \leq C\left(|\mathscr{H}(t)|+\left\|u_{t}\right\|_{L^{2}(\Omega)}^{2}+\rho_{q(\cdot)}(u)\right)$
(iii) $\|u\|_{q^{-}}^{s} \leq C\left(|\mathscr{H}(t)|+\left\|u_{t}\right\|_{L^{2}(\Omega)}^{2}+\|u\|_{q^{-}}^{q^{-}}\right)$

for any $u \in W_{0}^{1, p(\cdot)}(\Omega)$ and $\alpha p^{-} \leq s \leq \alpha q^{-}$.

Lemma 16. Assume that (12) and (15) hold. Then, the solution of (1) satisfies

$$
\mathrm{\varrho}_{q(\cdot)}(u) \geq C\|u\|_{q^{-}}^{q^{-}},
$$

for some $C>0$.

Proof. Let

$$
\begin{aligned}
& \Omega^{+}=\{x \in \Omega /|u| \geq 1\}, \\
& \Omega^{-}=\{x \in \Omega /|u|<1\} .
\end{aligned}
$$


We have

$$
\begin{aligned}
\varrho_{q(\cdot)}(u) & =\int_{\Omega^{+}}|u|^{q(x)} d x+\int_{\Omega^{-}}|u|^{q(x)} d x \\
& \geq \int_{\Omega^{+}}|u|^{q(x)} d x+c_{1}\left(\int_{\Omega^{-}}|u|^{q(x)} d x\right)^{q^{+} / q^{-}} .
\end{aligned}
$$

This implies

$$
c_{2}\left(\mathrm{\varrho}_{q(\cdot)}(u)\right)^{q^{-} / q^{+}}+\varrho_{q(\cdot)}(u) \geq\|u\|_{q^{-}}^{q^{-}}
$$

Now, given (52), (60) leads to

$$
\mathrm{\varrho}_{q(\cdot)}(u) \geq\left[1+c_{2}\left(\frac{q^{-}}{\sigma}\right)^{\left(q^{-} / q^{+}\right)-1}\right]^{-1}\|u\|_{q^{-}}^{q^{-}}
$$

Thus, (57) follows.

Lemma 17. Suppose that (12) holds, and $u$ is a solution of (1). Then,

$$
\mathrm{Q}_{r(\cdot)}(u) \leq C\left(\mathrm{Q}_{q(\cdot)}^{r^{-} / q^{-}}(u)+\mathrm{Q}_{q(\cdot)}^{r^{+} / q^{-}}(u)\right) .
$$

Proof.

$$
\begin{aligned}
\mathrm{\varrho}_{r(\cdot)}(u) & \leq \int_{\Omega^{-}}|u|^{r^{-}} d x+\int_{\Omega^{+}}|u|^{r^{+}} d x \\
& \leq C\left[\left(\int_{\Omega^{-}}|u|^{q^{-}} d x\right)^{r^{-} / q^{-}}+\left(\int_{\Omega^{+}}|u|^{q^{-}} d x\right)^{r^{+} / q^{-}}\right] \\
& \leq C\left(\|u\|_{q^{-}}^{r^{-}}+\|u\|_{q^{-}}^{r^{+}}\right) \leq C\left(\mathrm{Q}_{q(\cdot)}^{r^{-} / q^{-}}(u)+\mathrm{Q}_{q(\cdot)}^{r^{+} / q^{-}}(u)\right) .
\end{aligned}
$$

We set

$$
\mathscr{F}(t)=\mathscr{H}^{1-\lambda}(t)+\varepsilon\left|u, u_{t}\right|_{L^{2}(\Omega)},
$$

for $\varepsilon$ small, which will be specified later, and for

$$
0<\lambda \leq \min \left\{\frac{\alpha q^{-}-r^{+}}{q^{-}\left(r^{+}-1\right)}, \frac{q^{-}-2}{2 q^{-}}\right\} .
$$

Now, we are in a position to prove Theorem 10 .
Proof. We differentiate (64) and use the equation in (1) to get

$$
\begin{aligned}
\mathscr{F}_{t}(t)= & (1-\lambda) \mathscr{H}^{-\lambda}(t) \mathscr{H}_{t}(t)+\varepsilon\left(\left\|u_{t}\right\|_{L^{2}(\Omega)}^{2}+\left|u, u_{t t}\right|_{L^{2}(\Omega)}\right) \\
\geq & (1-\lambda) \mathscr{H}^{-\lambda}(t) \mathscr{H}_{t}(t) \\
& +\varepsilon\left(\left\|u_{t}\right\|_{L^{2}(\Omega)}^{2}-\mathscr{M}(\mathcal{N} u(t)) \mathrm{Q}_{p(\cdot)}(\nabla u)+c^{-} \mathrm{Q}_{q(\cdot)}(u)\right) \\
& -\varepsilon d^{+}\left|g\left(u_{t}\right), u\right|_{L^{2}(\Omega)} \geq(1-\lambda) \mathscr{H}^{-\lambda}(t) \mathscr{H}_{t}(t) \\
& +\varepsilon\left(\left\|u_{t}\right\|_{L^{2}(\Omega)}^{2}-\frac{m_{2}}{\left(p^{-}\right)^{\beta-1}} \mathrm{Q}_{p(\cdot)}^{\beta}(\nabla u)+c^{-} \mathrm{Q}_{q(\cdot)}(u)\right) \\
& -\varepsilon d^{+}\left|g\left(u_{t}\right), u\right|_{L^{2}(\Omega)} \geq(1-\lambda) \mathscr{H}^{-\lambda}(t) \mathscr{H}_{t}(t) \\
& +\varepsilon\left(\left\|u_{t}\right\|_{L^{2}(\Omega)}^{2}-\frac{m_{2}}{\left(p^{-}\right)^{\beta-1}} \mathrm{Q}_{p(\cdot)}^{\alpha}(\nabla u)+c^{-} \mathrm{Q}_{q(\cdot)}(u)\right) \\
& -\varepsilon d^{+}\left|g\left(u_{t}\right), u\right|_{L^{2}(\Omega)} \cdot
\end{aligned}
$$

Adding and subtracting the term $\varepsilon(1-\eta) q^{-} \mathscr{H}(t)$, for $0<\eta<1$, from the right-hand side of (56), by using (49) and (10), we get

$$
\begin{aligned}
\mathscr{F}_{t}(t) \geq & (1-\lambda) \mathscr{H}^{-\lambda}(t) \mathscr{H}_{t}(t)+\varepsilon(1-\eta) q^{-} \mathscr{H}(t)-\varepsilon d^{+}\left|g\left(u_{t}\right), u\right|_{L^{2}(\Omega)} \\
& +\varepsilon\left(\left\|u_{t}\right\|_{L^{2}(\Omega)}^{2}-\frac{m_{2}}{\left(p^{-}\right)^{\beta-1}} \mathrm{\varrho}_{p(\cdot)}^{\alpha}(\nabla u)+c^{-} \mathrm{\varrho}_{q(\cdot)}(u)\right) \\
& +\varepsilon(1-\eta) q^{-}\left(-\mathscr{E}_{1}+\frac{1}{2}\left\|u_{t}\right\|_{L^{2}(\Omega)}^{2}+\widehat{M}(\mathscr{N} u(t))-\frac{c^{-}}{q^{-}} \mathrm{Q}_{q(\cdot)}(u)\right) \\
\geq & (1-\lambda) \mathscr{H}^{-\lambda}(t) \mathscr{H}_{t}(t)+\varepsilon(1-\eta) q^{-} \mathscr{H}(t)-\varepsilon d^{+}\left|g\left(u_{t}\right), u\right|_{L^{2}(\Omega)} \\
& +\varepsilon\left(\left\|u_{t}\right\|_{L^{2}(\Omega)}^{2}-\frac{m_{2}}{\left(p^{-}\right)^{\beta-1}} \rho_{p(\cdot)}^{\alpha}(\nabla u)+c^{-} \mathrm{\varrho}_{q(\cdot)}(u)\right) \\
& +\varepsilon(1-\eta) q^{-}\left(\left(-\frac{\mathscr{E}_{1} c^{+}}{q^{-} \kappa}-\frac{c^{-}}{q^{-}}\right) \mathrm{\varrho}_{q(\cdot)}(u)+\frac{1}{2}\left\|u_{t}\right\|_{L^{2}(\Omega)}^{2}\right) \\
& +\varepsilon(1-\eta) q^{-}\left(\frac{m_{1}}{\alpha\left(p^{+}\right)^{\alpha}} \mathrm{\varrho}_{p(\cdot)}^{\alpha}(\nabla u)\right) .
\end{aligned}
$$

Then, for $\eta$ small enough, we have

$$
\begin{aligned}
\mathscr{F}_{t}(t) \geq & (1-\lambda) \mathscr{H}^{-\lambda}(t) \mathscr{H}_{t}(t)-\varepsilon d^{+}\left|g\left(u_{t}\right), u\right|_{L^{2}(\Omega)} \\
& +\varepsilon \delta\left(\mathscr{H}(t)+\left\|u_{t}\right\|_{L^{2}(\Omega)}^{2}+\varrho_{p(\cdot)}^{\alpha}(\nabla u)+\varrho_{q(\cdot)}(u)\right),
\end{aligned}
$$

where

$$
\begin{aligned}
\delta= & \min \left\{1-\eta q^{-}, 1+\frac{(1-\eta) q^{-}}{2}, \frac{(1-\eta) q^{-} m_{1}}{\alpha\left(p^{+}\right)^{\alpha}}\right. \\
& \left.-\frac{m_{2}}{\left(p^{-}\right)^{\beta-1}}, \eta c^{-}-\frac{(1-\eta) \mathscr{E}_{1} c^{+}}{\kappa}\right\} .
\end{aligned}
$$


Recall Young's inequality

$$
X Y \leq \frac{\delta_{1} X^{l_{1}}}{l_{1}}+\frac{\delta_{1}^{-l_{2}} Y^{l_{2}}}{l_{2}}
$$

where $X, Y \geq 0, \delta_{1}>0, l_{1}, l_{2} \in \mathbb{R}^{+}$, such that $\left(1 / l_{1}\right)+\left(1 / l_{2}\right)$ $=1$. Applying $(70)$ to estimate the term $\left|g\left(u_{t}\right), u\right|_{L^{2}(\Omega)}$, we get

$$
\int_{\Omega}\left|u_{t}\right|^{r(x)-1}|u| d x \leq \frac{1}{r^{-}} \int_{\Omega} \delta_{1}^{r(x)}|u|^{r(x)} d x+\frac{r^{+}-1}{r^{+}} \int_{\Omega} \delta_{1}^{-r(x) /(r(x)-1)}\left|u_{t}\right|^{r(x)} d x,
$$

where

$$
\delta_{1}^{-r(x) /(r(x)-1)}=\xi \mathscr{H}^{-\lambda}(t)
$$

where $\xi$ is a large constant to be specified later.

Now, by using (32) and (49), we get

$\mathrm{\varrho}_{r(\cdot)}\left(u_{t}\right) \leq \frac{\mathscr{H}_{t}(t)}{d^{+}}-\frac{\tilde{\sigma}}{q^{+} d^{+}} \varrho_{q(\cdot)}(u) \leq \frac{\mathscr{H}_{t}(t)}{d^{+}}-\frac{\tilde{\sigma} \kappa}{q^{+} d^{+}} \leq \frac{\mathscr{H}_{t}(t)}{d^{+}}$

Combining (73) and (71) yields

$$
\begin{aligned}
& \int_{\Omega}\left|u_{t}\right|^{r(x)-1}|u| d x \leq \frac{1}{r^{-}} \int_{\Omega} \xi^{1-r(x)}|u|^{r(x)} \mathscr{H}^{\lambda(r(x)-1)}(t) d \\
& +\frac{\left(r^{+}-1\right) \xi}{r^{+} d^{+}} \mathscr{H}^{-\lambda}(t) \mathscr{H}_{t}(t) .
\end{aligned}
$$

Substituting (74) in (68), we obtain

$$
\begin{aligned}
\mathscr{F}_{t}(t) \geq & {\left[(1-\lambda)-\varepsilon \frac{\left(r^{+}-1\right) \xi}{r^{+}}\right] \mathscr{H}^{-\lambda}(t) \mathscr{H}_{t}(t) } \\
& +\varepsilon \delta\left(\mathscr{H}(t)+\left\|u_{t}\right\|_{L^{2}(\Omega)}^{2}+\varrho_{p(\cdot)}^{\alpha}(\nabla u)+\varrho_{q(\cdot)}(u)\right) \\
& -\varepsilon \frac{\xi^{1-r^{-}} d^{+}}{r^{-}} C_{1} \mathscr{H}^{\lambda\left(r^{+}-1\right)}(t) \varrho_{r(\cdot)}(u) .
\end{aligned}
$$

To estimate the last term in (75), we use (62) and (52) to get

$$
\mathscr{H}^{\lambda\left(r^{+}-1\right)}(t) \mathrm{Q}_{r(\cdot)}(u) \leq C\left(\mathrm{\varrho}_{q(\cdot)}^{\left(r^{-} / q^{-}\right)+\lambda\left(r^{+}-1\right)}(u)+\mathrm{\varrho}_{q(\cdot)}^{\left(r^{+} / q^{-}\right)+\lambda\left(r^{+}-1\right)}(u)\right) .
$$

Then, we use (65) and Lemma 9, for

$$
\begin{aligned}
& s=r^{+}+\lambda q^{-}\left(r^{+}-1\right) \leq \alpha q^{-}, \\
& s=r^{-}+\lambda q^{-}\left(r^{+}-1\right) \leq \alpha q^{-},
\end{aligned}
$$

to deduce from (76) that

$$
\mathscr{H}^{\lambda\left(r^{+}-1\right)}(t) \mathrm{\varrho}_{r(\cdot)}(u) \leq C\left(\|\nabla u\|_{p(\cdot)}^{\alpha p^{-}}+\mathrm{\varrho}_{q(\cdot)}(u)\right) .
$$

By exploiting Lemmas 5 and 12, we get

$$
\mathrm{Q}_{p(\cdot)}^{\alpha}(\nabla u) \geq C\|\nabla u\|_{p(\cdot)}^{\alpha p^{-}}
$$

Combining (75), (78), and (79) leads to

$$
\begin{aligned}
& \mathscr{F}_{t}(t) \geq {\left[(1-\lambda)-\varepsilon \frac{\left(r^{+}-1\right) \xi}{r^{+}}\right] \mathscr{H}^{-\lambda}(t) \mathscr{H}_{t}(t)+\varepsilon\left(\delta-\frac{\xi^{1-r^{-}} d^{+}}{r^{-}} C\right) } \\
& \cdot\left[\mathscr{H}(t)+\left\|u_{t}\right\|_{L^{2}(\Omega)}^{2}+\|\nabla u\|_{p(\cdot)}^{\alpha p^{-}}+\mathrm{Q}_{q(\cdot)}(u)\right] .
\end{aligned}
$$

Now, we pick $\xi$ large enough and $\varepsilon$ so small such that

$$
\begin{aligned}
\delta_{2} & =\delta-\frac{\xi^{1-r^{-}} d^{+}}{r^{-}} C>0, \\
\varepsilon & \leq \frac{(1-\lambda) r^{+}}{\xi\left(r^{+}-1\right)}, \\
\mathscr{F}(0) & =\mathscr{H}^{1-\lambda}(0)+\varepsilon\left|u_{0}, u_{1}\right|_{L^{2}(\Omega)}>0 .
\end{aligned}
$$

Then, by using (57), (80) takes the form

$$
\mathscr{F}_{t}(t) \geq \delta_{2} \varepsilon\left[\mathscr{H}(t)+\left\|u_{t}\right\|_{L^{2}(\Omega)}^{2}+\|\nabla u\|_{p(\cdot)}^{\alpha p^{-}}+\|u\|_{q^{-}}^{q^{-}}\right] .
$$

Therefore, we get

$$
\mathscr{F}(t) \geq \mathscr{F}(0)>0, \quad \text { for all } t \geq 0
$$

On the other hand, the application of the Hölder inequality yields

$$
|| u,\left.\left.u_{t}\right|_{L^{2}(\Omega)}\right|^{1-\lambda} \leq C\|u\|_{q^{-}}^{1 /(1-\lambda)}\left\|u_{t}\right\|_{L^{2}(\Omega)}^{1 /(1-\lambda)},
$$

and from (70), we get

$$
|| u,\left.\left.u_{t}\right|_{L^{2}(\Omega)}\right|^{1-\lambda} \leq C\left[\|u\|_{q^{-}}^{\theta_{1} /(1-\lambda)}+\left\|u_{t}\right\|_{L^{2}(\Omega)}^{\theta_{2} /(1-\lambda)}\right]
$$

for $\left(1 / \theta_{1}\right)+\left(1 / \theta_{2}\right)=1$. Setting $\theta_{1} /(1-\lambda)=(2 /(1-2 \lambda)) \leq q^{-}$, we get $\theta_{2}=2(1-\lambda)$ by virtue of (65). Therefore, (85) takes the form

$$
|| u,\left.\left.u_{t}\right|_{L^{2}(\Omega)}\right|^{1-\lambda} \leq C\left[\|u\|_{q^{-}}^{s}+\left\|u_{t}\right\|_{L^{2}(\Omega)}^{2}\right]
$$

where $s=2 /(1-2 \lambda)$. By recalling Corollary 15 , we get

$$
|| u,\left.\left.u_{t}\right|_{L^{2}(\Omega)}\right|^{1-\lambda} \leq C\left(|\mathscr{H}(t)|+\left\|u_{t}\right\|_{L^{2}(\Omega)}^{2}+\|u\|_{q^{-}}^{q^{-}}\right) .
$$


Now, (87) and the following Minkowski's inequality

$$
(X+Y)^{i} \leq 2^{i-1}\left(X^{i}+Y^{i}\right)
$$

will give

$$
\begin{aligned}
\mathscr{F}^{1 /(1-\lambda)}(t) & =\left[\mathscr{H}^{1-\lambda}(t)+\varepsilon\left|u, u_{t}\right|_{L^{2}(\Omega)}\right]^{1 /(1-\lambda)} \\
& \leq 2^{\lambda /(1-\lambda)}\left(\mathscr{H}(t)+\varepsilon^{1 /(1-\lambda)}|| u,\left.\left.u_{t}\right|_{L^{2}(\Omega)}\right|^{1-\lambda}\right) \\
& \leq C\left(|\mathscr{H}(t)|+\left\|u_{t}\right\|_{L^{2}(\Omega)}^{2}+\|u\|_{q^{-}}^{q^{-}}\right) \\
& \leq C\left(\mathscr{H}(t)+\left\|u_{t}\right\|_{L^{2}(\Omega)}^{2}+\|\nabla u\|_{p(\cdot)}^{\alpha p^{-}}+\|u\|_{q^{-}}^{q^{-}}\right) .
\end{aligned}
$$

By combining (82) and (89), we obtain

$$
\mathscr{F}_{t}(t) \geq \mu \mathscr{F}(t)^{1 /(1-\lambda)}(t),
$$

where $\mu$ is a positive constant. A simple integration of (90) over $(0, t)$ yields

$$
\mathscr{F}^{1 /(1-\lambda)}(t) \geq \frac{1}{\mathscr{F}^{-\lambda /(1-\lambda)}(0)-(\mu \lambda t /(1-\lambda))},
$$

which implies that the solution blows up in finite time $T^{*}$, such that

$$
T^{*} \leq \frac{1-\lambda}{\mu \lambda \mathscr{F}^{\lambda /(1-\lambda)}(0)}
$$

This completes the proof of Theorem 10 .

\section{Proof of Theorem 11}

We set

$$
\mathscr{H}(t):=-\mathscr{E}(t)
$$
lemma.

To prove our main result, we first establish the following

Lemma 18. Let $u$ be the solution of (1). Then, there exists a constant $C>0$ such that

$$
\|\nabla u(t, x)\|_{p(\cdot)} \geq C, \quad \forall t \geq 0
$$

Proof. Suppose that, by contradiction, there exists a sequence $t_{k}$ such that

$$
\|\nabla u(t, x)\|_{p(\cdot)} \longrightarrow 0, \quad \text { as } k \longrightarrow \infty
$$

Then, by using Lemmas 2 and 5, we get

$$
\lim _{k \rightarrow \infty} \mathscr{E}\left(t_{k}\right) \geq 0
$$

which contradicts the fact that $\mathscr{E}(t)<0, \forall t \geq 0$.

Using (93) and (94) and applying the same procedures used to prove Theorem 10 will give the proof of Theorem 11 .

\section{Data Availability}

No data were used in this study.

\section{Conflicts of Interest}

The authors declare that they have no competing interests.

\section{Authors' Contributions}

The authors contributed equally and significantly in writing this paper. All authors read and approved the final manuscript.

\section{References}

[1] G. Kirchhoff and K. Hensel, Vorlesungen über mathematische Physik, 1883.

[2] K. Ono, "Global existence, decay, and blowup of solutions for some mildly degenerate nonlinear Kirchhoff strings," Journal of differential equations, vol. 137, no. 2, pp. 273-301, 1997.

[3] S.-T. Wu and L.-Y. Tsai, "Blow-up of solutions for some nonlinear wave equations of Kirchhoff type with some dissipation," Nonlinear Analysis: Theory, Methods \& Applications, vol. 65, no. 2, pp. 243-264, 2006.

[4] T. Matsuyama and R. Ikehata, "On global solutions and energy decay for the wave equations of Kirchhoff type with nonlinear damping terms," Journal of Mathematical Analysis and Applications, vol. 204, no. 3, pp. 729-753, 1996.

[5] J. M. Ball, "Remarks on blow-up and nonexistence theorems for nonlinear evolution equations," The Quarterly Journal of Mathematics, vol. 28, no. 4, pp. 473-486, 1977.

[6] V. A. Galaktionov and S. I. Pohozaev, "Blow-up and critical exponents for nonlinear hyperbolic equations," Nonlinear Analysis: Theory, Methods \& Applications, vol. 53, no. 3-4, pp. 453-466, 2003.

[7] H. A. Levine, "Some additional remarks on the nonexistence of global solutions to nonlinear wave equations," SIAM Journal on Mathematical Analysis, vol. 5, no. 1, pp. 138-146, 1974.

[8] S. Antontsev, "Equation des ondes avec $\mathrm{p}(\mathrm{x}, \mathrm{t})$-Laplacian et un terme dissipatif : blow-up des solutions," Comptes Rendus Mécanique, vol. 339, no. 12, pp. 751-755, 2011.

[9] S. Boulaaras, A. Draifia, and K. Zennir, "General decay of nonlinear viscoelastic Kirchhoff equation with Balakrishnan-Taylor damping and logarithmic nonlinearity," Mathematical Methods in the Applied Sciences, vol. 42, no. 14, pp. 4795-4814, 2019.

[10] J. Ferreira and S. A. Messaoudi, "On the general decay of a nonlinear viscoelastic plate equation with a strong damping and $\overrightarrow{\mathrm{p}}(\mathrm{x}, \mathrm{t})$-Laplacian," Nonlinear Analysis: Theory, Methods \& Applications, vol. 104, pp. 40-49, 2014.

[11] L. Sun, Y. Ren, and W. Gao, "Lower and upper bounds for the blow-up time for nonlinear wave equation with variable 
sources," Computers \& Mathematics with Applications, vol. 71, no. 1, pp. 267-277, 2016.

[12] Z. Tebba, S. Boulaaras, H. Degaichia, and A. Allahem, "Existence and blow-up of a new class of nonlinear damped wave equation," Journal of Intelligent \& Fuzzy Systems, vol. 38, no. 3, pp. 2649-2660, 2020.

[13] K. Zennir, "Stabilization for solutions of plate equation with time-varying delay and weak-viscoelasticity in $\mathrm{R}^{\mathrm{n}}$," Russian Mathematics, vol. 64, no. 9, pp. 21-33, 2020.

[14] E. Piskin, "Finite time blow up of solutions of the Kirchhofftype equation with variable exponents," International Journal of Nonlinear Analysis and Applications, vol. 11, no. 1, pp. 3745, 2020.

[15] B. Feng and H. Li, "Energy decay for a viscoelastic Kirchhoff plate equation with a delay term," Boundary Value Problems, vol. 2016, no. 1, 16 pages, 2016.

[16] K. Zennir, M. Bayoud, and G. Svetlin, "Decay of solution for degenerate wave equation of Kirchhoff type in viscoelasticity," International Journal of Applied and Computational Mathematics, vol. 4, no. 1, pp. 1-18, 2018.

[17] J. Musielak, Orlicz Spaces and Modular Spaces, Lecture Notes in Mathematics, vol. 1034, pp. 1-216, 1983.

[18] X. Fan and D. Zhao, "On the spaces $\mathrm{L}^{\mathrm{p}}(\mathrm{x})(\mathrm{O})$ and $\mathrm{W}^{(\mathrm{m}, \mathrm{p}(\mathrm{x}))}$ (O)," The Journal of Mathematical Analysis and Applications, vol. 263, no. 2, pp. 424-446, 2001.

[19] X. Fan, J. Shen, and D. Zhao, "Sobolev embedding theorems for spaces _ $\mathrm{W}^{\mathrm{k}, \mathrm{p}(\mathrm{x})}(\Omega)$," The Journal of Mathematical Analysis and Applications, vol. 262, no. 2, pp. 749-760, 2001. 